\title{
A computational model for the prediction of total knee replacement kinematics in the sagittal plane
}

\author{
A.-C. Godest ${ }^{\mathrm{a}}$, C. Simonis de Cloke ${ }^{\mathrm{b}}$, M. Taylor ${ }^{\mathrm{b}}$, P.J. Gregson ${ }^{\mathrm{b}, *}$, \\ A.J. Keane ${ }^{\mathrm{b}}$, S. Sathasivan ${ }^{\mathrm{c}}$, P.S. Walker ${ }^{\mathrm{c}}$ \\ "Engineering Materials Department, University of Southampton Highfield, Southampton, SO17 IBJ, UK \\ 'Mechanical Engineering Department, University of Southampton Highfield, Southampton, SO17 IBJ, UK \\ ${ }^{\circ}$ Centre for Biomedical Engineering, University College London, Royal National Orthopaedic Hospital Trust, Stanmore, Middlesex HA7 4LP, UK \\ Accepted 12 October 1999
}

\begin{abstract}
A computational model has been developed using a current generation computer-aided engineering (CAE) package to predict total knee replacement (TKR) kinematic in the sagittal plane. The model includes friction and soft tissue restraint varying according to the flexion angle. The model was validated by comparing the outcomes of anterior-posterior (A-P) laxity tests of two contemporary knee replacements against data obtained from a knee simulating machine. It was also validated against predictions from a computer model reported in the literature. Results show good agreement in terms of A-P displacements. Further tests were performed to determined the influence of the soft tissue restraints varying with flexion angle. This work represents the first attempt to use a sophisticated commercial CAE package to predict TKR motions and the advantages of the modelling procedure chosen are discussed. (C) 2000 Elsevier Science Ltd. All rights reserved.
\end{abstract}

Keywords: 2D knee kinematics; Knee replacement; Computational knee modelling; Knee laxity; Virtual prototyping software

\section{Introduction}

Total knee replacement has become a routine surgical procedure to relieve pain, restore alignment and to restore knee function. Even if, for some designs, survival rates of better then $90 \%$ have been achieved at 10 years (Knutson et al., 1994), three types of TKR failure have been reported, (i) failure to reproduce normal knee function (Luger et al., 1997), (ii) bone-implant interface failure leading to implant loosening and (iii) wear of the prosthesis itself (Blunn et al., 1997). These three types of failure are clearly related to the motions and loads occurring at the bearing surfaces of the prosthesis components. Motions and loads govern in particular the implant wear process which are an important concern as performance requirements (both load level and lifetime) of TKR implants increase.

Determination of knee prosthesis kinematics during function is therefore important when addressing these

\footnotetext{
* Corresponding author. Tel.: + 44-1703-593760; fax: + 44-1703593016.

E-mail address: pjg5@soton.ac.uk (P.J. Gregson)
}

problems. Computer models can be useful tools for predicting TKR kinematics and evaluating the mechanical behaviour of the prosthesis components as a function of their geometry. Three-dimensional computer models have been developed to predict TKR kinematics and have provided indications on how TKR designs, ligament restraints or surface friction influence the joint motions (Essinger et al., 1989; Garg and Walker, 1990; Sathasivam and Walker, 1997). However, the models reported in the literature all required a significant amount of specific programming. Increasing the accuracy and complexity of such models would require longer computer programs. Such models may also be difficult to transfer between researchers. From a design point of view, the necessity to reprogram for each design iteration make these cumbersome tools. None of the TKR models reported in the literature were developed using computer assisted engineering packages (CAE). CAE packages now make it possible to perform rapid calculations and to simulate motions of complex systems without requiring any programming. Widely used in the automotive and aircraft industries, suitably customised computer packages could also be useful in the biomechanics field. Computer models developed on a current generation CAE 

package could be used to evaluate the influence of TKR design on knee joint kinematics, reducing design time and increasing the ability for pre-prototype evaluation of designs. I-DEAS ${ }^{\mathrm{TM}}$ Master Series and $\mathrm{ADAMS}^{\mathrm{TM}}$ kinematic solver are typical of modern CAE packages. In order to asses their adaptability to biomechanics matters, a simple model was developed to predict TKR kinematics in the sagittal plane. The model was based on the mechanical arrangement used in the Stanmore knee simulator (Walker et al., 1997) and in the model developed by Sathasivam and Walker (1997). In order to validate the model, a series of anterior-posterior laxity tests was performed on two prostheses, for which experimental and numerical data are available (Walker et al., 1997; Sathasivam and Walker, 1997). The software was then used to improve the representation of the soft tissue restraints described by Sathasivam and Walker (1997).

\section{Methods and materials}

\subsection{Description of the model}

The kinematics of the Stanmore knee simulator developed by Walker et al. (1997) were modelled. The Stanmore knee simulator allows six degrees of freedom, the three translations and the three rotations of the knee joint. However, as a first attempt to evaluate CAE package capabilities, the modelling was restricted to predicting TKR motions in the sagittal plane. Thus, only three motions were allowed between the components. The femoral component was allowed to translate in the proximal-distal direction and to rotate about its transverse axis (Fig. 1), which was determined from previous experi- ments (Sathasivam and Walker, 1997 or Walker et 1997). The tibial component was allowed to translat the anterior-posterior direction (Fig. 1).

The model was quasi-static. The principal loads br by the knee joint in the sagittal plane are a compres force $\left(F_{\mathrm{c}}\right)$ applied to the femoral component, paralls the tibia fixed axis, and an anterior-posterior force ( applied to the tibial component, as presented in Fig. order to conform with the mechanical arrangemer Stanmore simulator, the anterior-posterior motion o tibial tray relative to the femoral component was strained by two non-linear springs (Fig. 1). The ante non-linear spring represents the action of the soft tis restraining the anterior displacement of the tibia relc to the femur (Crowninshield et al., 1976). The postt non-linear spring represents the action of soft tis restraining the posterior displacement of the tibia reli to the femur (Crowninshield et al., 1976). The f exerted by the anterior and posterior bumpers in Stanmore simulator are defined by

$$
\begin{aligned}
& F_{\mathrm{a}}=K_{1} x^{2}+k_{2} x=0.444 x^{2}+2.334 x, \\
& F_{\mathrm{p}}=K_{3} x^{2}+k_{4} x=3.910 x^{2}+7.960 x,
\end{aligned}
$$

where $F_{\mathrm{a}}$ and $F_{\mathrm{p}}$ are the forces exerted, respectively the anterior and posterior springs, $x$ is the compres displacement of each spring, and $k_{1}, k_{3}$ and $k_{2} k_{4}$ arr stiffness constants of the springs, respectively, Wher veloped by Sathasivam and Walker (1997), these e tions did not vary with flexion angle.

Due to the effect of friction, TKR motions cal unsmooth in contrast with the situation in a natural ] where the friction is so small as to be insignificant predict TKR kinematics in a realistic way, friction incorporated within the model.
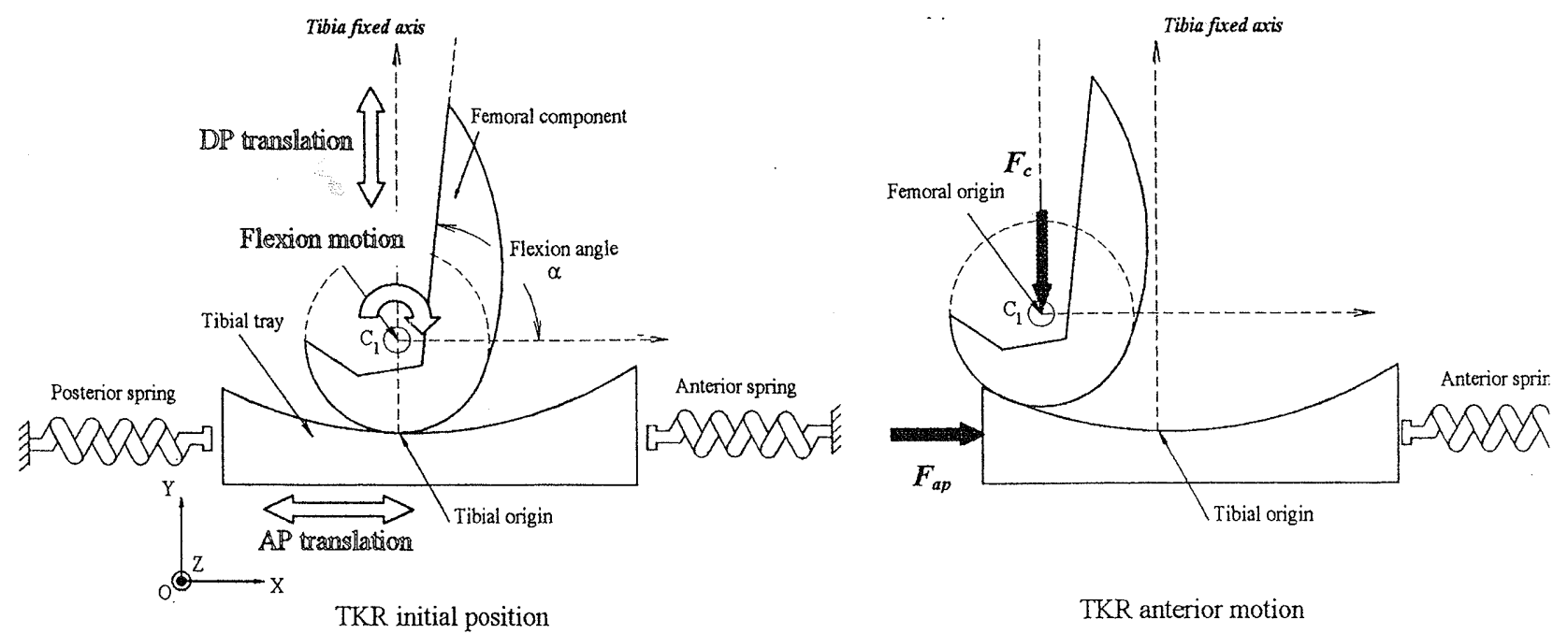

TKR anterior motion

Fig. 1. Discription of the TKR motions in the sagittal plane when subject to a compressive force $F_{\mathrm{c}}$ and an anterior shear force $F_{\mathrm{ap}}$. 



\subsection{Computer modelling}

I-DEAS ${ }^{\mathrm{TM}}$ is an integrated package of mechanical engineering tools and was used throughout this study. This software is designed to facilitate a concurrent engineering approach to mechanical engineering product design and analysis. Kinematic analyses can be carried out by creating a mechanism and solving its motions using the integrated $\mathrm{ADAMS}^{\mathrm{TM}}$ kinematic solver. For a particular load case, the solver calculates the motions of the mechanism and forces as a function of time. The successive positions of the rigid bodies are displayed in successive configurations of the mechanism or frames.

To analyse the relative motions between the main components of a TKR implant under different loading conditions, a solid model was built to represent the Stanmore simulator. The mechanism contains nine rigid bodies, four to model the TKR components and their contacting profiles and five to build the grounded frame holding the TKR components (Fig. 2). Joints and constraints were incorporated into the assembly to restrict the relative motions between the components of the mechanism. The femoral component was allowed to rotate about its transverse axis but this flexion motion was controlled in order to simulate any desired activity. The flexion angle of the joint was thus chosen and defined in the initial configuration of the mechanism. The femoral component is rotated about its transverse axis (axis of the flexion bar) from its $0^{\circ}$ flexion position to a chosen angle $\alpha$ (Fig. 1). The lowest point of the lower femoral surface was positioned to be in contact with the lowest point of the tibial upper surface. Additionally, the femoral component was allowed to translate in the proximal-distal direction relative to the back wall using a translational joint. The tibial tray was allowed to translate in the anterior-posterior direction relative to the bottom wall using another translational joint. The femoral and tibial

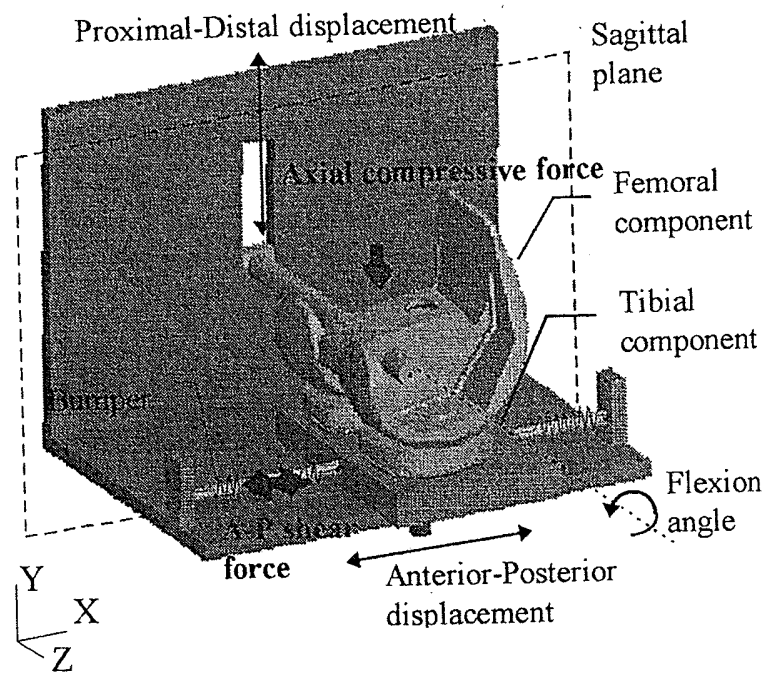

Fig. 2. I-DEAS ${ }^{\mathrm{TM}}$ total knee replacement computational model. components were constrained to remain in contact along their sagittal profiles using a cam-cam joint. The cam-cam constraint restricts a planar curve of one rigid body to be in contact and tangential to a planar curve of a second rigid body.

Non-linear springs were used to define explicitly the forces exerted by the springs as shown in Eqs. (1) and (2). Springs forces $F_{\mathrm{a}}$ and $F_{\mathrm{p}}$ are defined by two negative functions. This indicates to the kinematic solver that these two forces act against the tibial tray displacements. They restrain the anterior-posterior motion of the tibial tray in the same way as two non-linear springs working only in compression.

To incorporate friction, the friction force $(f)$ was decomposed into its horizontal $\left(f_{x}\right)$ and vertical $\left(f_{y}\right)$ components and applied directly to the mechanism. To ensure that the frictional force $f$ opposes the prevailing motion, the horizontal component $f_{x}$ is applied in the anterior direction when $F_{\text {ap }}$ is an anterior force, and in the posterior direction when $F_{\text {ap }}$ is a posterior force. The vertical frictional component $f_{y}$ is always applied downwards for any motion.

The mechanism was subjected to four external forces, the compressive force $F_{\mathrm{c}}$ applied to the femoral component, the anterior-posterior force $F_{\text {ap }}$ applied to the tibial tray, the force modelling soft tissue restraints and the friction force. Since the kinematic solver does not solve mechanisms only driven by forces, a constant velocity translational motion was applied to the tibial tray, either in the anterior or posterior direction. At each time step, the kinematic solver displays the relative positions of the TKR components and calculates the reaction forces within the mechanism.

\subsection{Validation studies}

Anterior-posterior laxity tests were carried out using the same loading conditions, soft tissue restraints, with and without friction by Walker et al., 1997; Sathasivam and Walker, 1997). In order to validate the model, the two TKR designs tested previously by Walker et al. (1997), the Kinemax Lowstress and the Kinemax Condylar TKR from Howmedica, were tested at flexion angles of 10 and $60^{\circ}$. A constant compressive force $F_{\mathrm{c}}$ of $1500 \mathrm{~N}$ and an anterior-posterior force $F_{\text {ap }}$ ranging from $-300 \mathrm{~N}$ to $+300 \mathrm{~N}$ were applied. Friction was incorporated into the model using static and kinematic coefficients of friction of 0.07 (Sathasivam and Walker, 1997).

\subsection{Soft tissue constraint studies}

In previous studies (Sathasivam and Walker, 1997; Luger et al., 1997), soft tissues were modelled as non-linear springs whose stiffness did not vary with the flexion angle (as presented in the validation results). However, in the intact knee, soft tissue restraints vary significantly with 

flexion angles (Fukubayashi et al., 1982). In order to take into account such variations, alternative values for the constant $k_{1}, k_{2}, k_{3}$ and $k_{4}$ were calculated based on Fukubayashi's data (1982) and tested at 10 and $60^{\circ}$ flexion (Table 1). The influence of varying soft tissue

Table 1

Spring stiffnesses $k_{1}, k_{3}\left(\mathrm{~N} / \mathrm{mm}^{2}\right)$ and $k_{2}, k_{4}(\mathrm{~N} / \mathrm{mm})$ for varying flexion angles

\begin{tabular}{llllll}
\hline TKR type & $\begin{array}{l}\text { Flexion } \\
\text { angles }\end{array}$ & $k_{1}$ & $k_{2}$ & $k_{3}$ & $k_{4}$ \\
\hline $\begin{array}{l}\text { Cruciate retaining } \\
\text { TKR kinemax } \\
\text { lowstress, Kinemax } \\
\text { condylar }\end{array}$ & $10^{\circ}$ & 0.44 & 0 & 6.76 & 0 \\
\hline
\end{tabular}

restraints with flexion angle on the anterior-post laxity of the two TKR designs was determined.

\section{Results}

\subsection{Validation}

Figs. 3 and 4 show the prediction at 10 and $60^{\circ}$ fle for the Kinemax Lowstress and Kinemax Condylar designs, with friction and no friction, compared wit predictions of the Sathasivam model (Sathasivam Walker, 1997). For cases with and without friction force-displacement curves matched Sathasivan resul both 10 and $60^{\circ}$ flexion.

Fig. 5 shows the validation results against data the knee simulating machine developed by Walker (1997). Tests carried out with the knee simulator

$10^{\circ}$ flexion

\section{NO FRICTION}

\section{$60^{\circ}$ flexion}
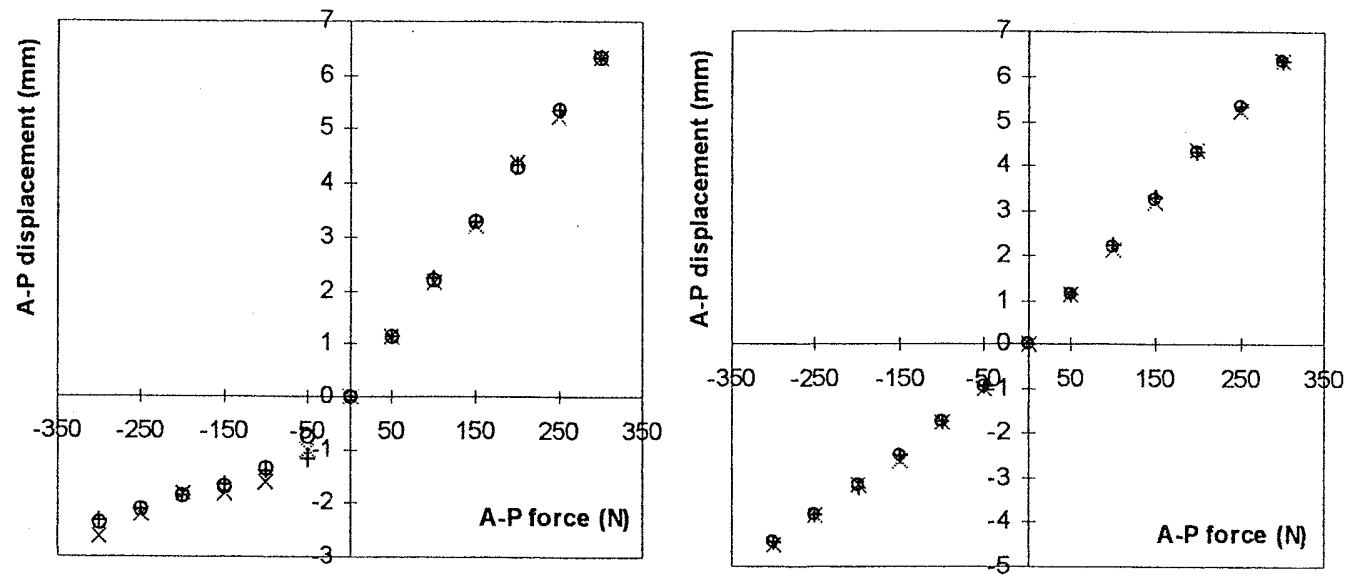

FRICTION $(\mu=0.07)$
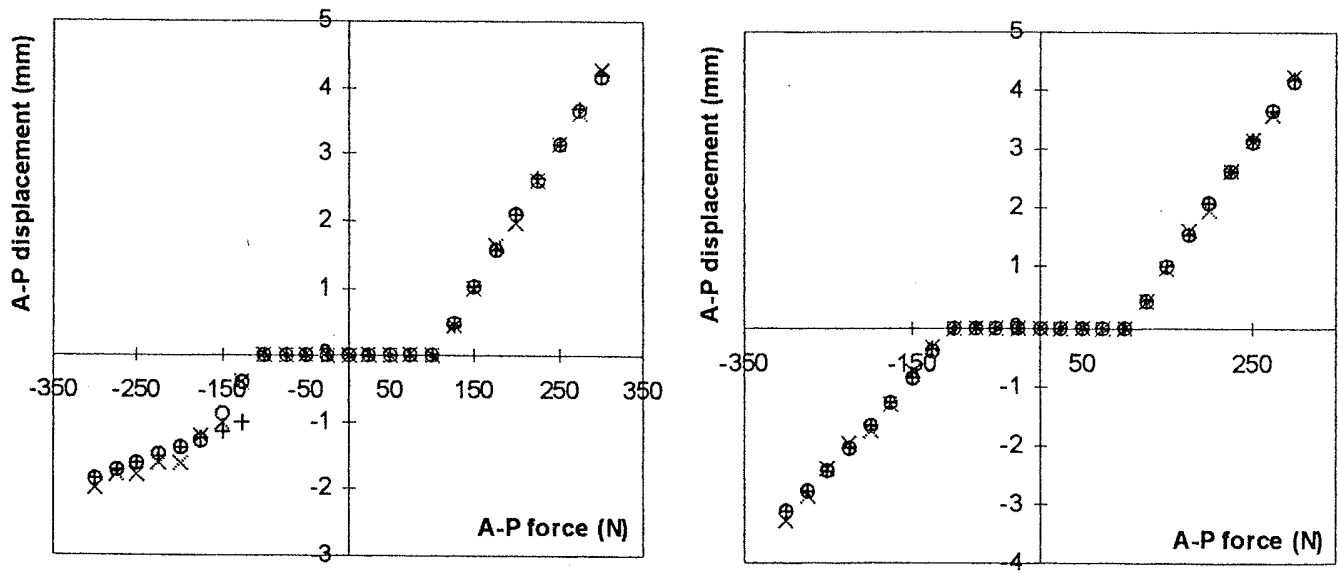

Fig. 3. A-P laxity results for the Kinemax Lowstress TKR at 10 and $60^{\circ}$ flexion, predicted by I-DEAS ${ }^{\mathrm{TM}}(\oplus)$ and by Sathasivan mode (Sathasivam and Walker, 1997). 



\section{$10^{\circ}$ flexion}

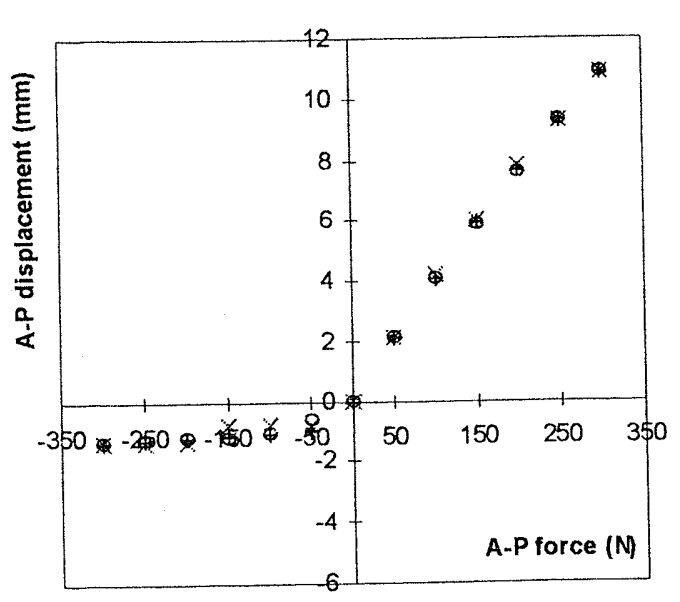

\section{$60^{\circ}$ flexion}

\section{NO FRICTION}

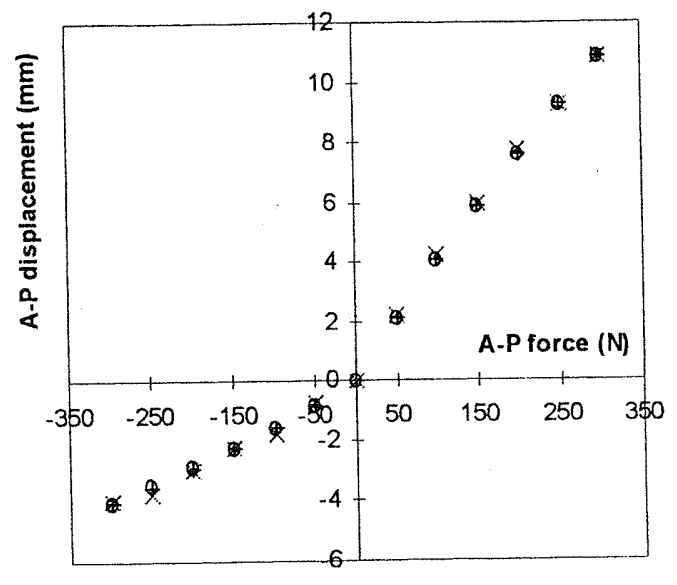

FRICTION $(\mu=0.07)$
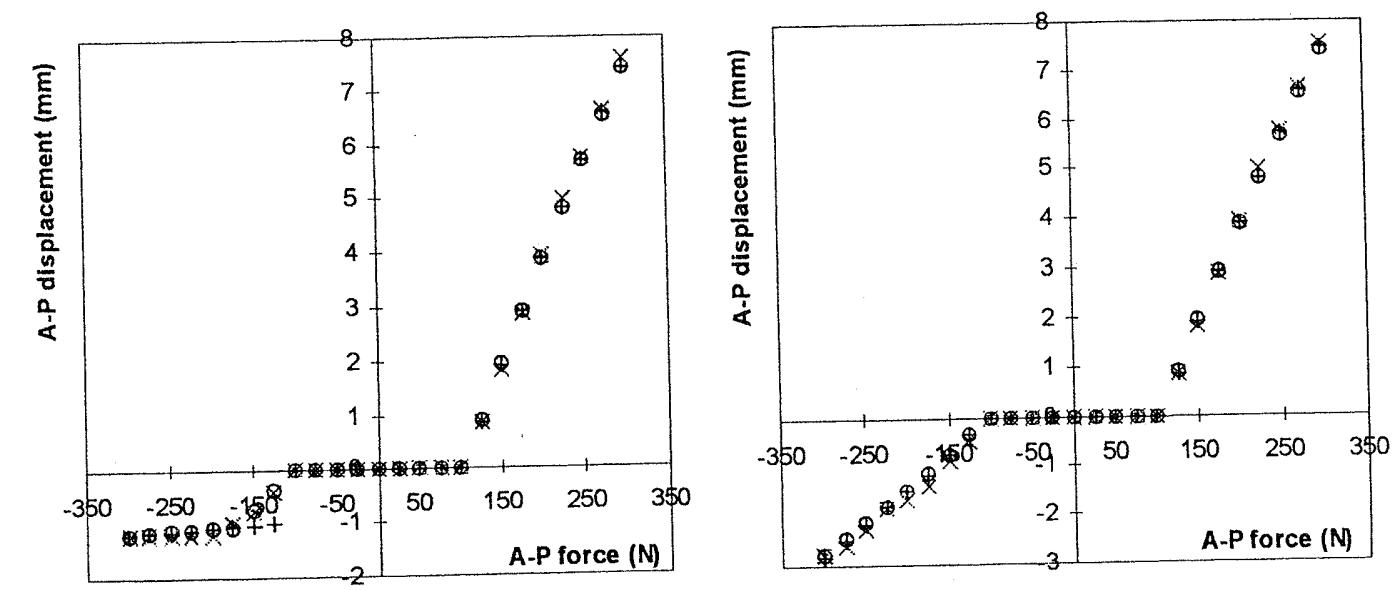

Fig. 4. A-P laxity results for the Kinemax Condylar TKR at 10 and $60^{\circ}$ flexion, predicted by $\operatorname{I-DEAS}^{\mathrm{TM}}(\oplus)$ and by Sathasivam model $(\times)$ (Sathasivam and Walker, 1997).

cyclic and the anterior shear force had a maximum magnitude of 250 instead of $300 \mathrm{~N}$. Similarities in the data between the model predictions and the cyclic tests were expected when the anterior force is increased from 0 to $250 \mathrm{~N}$ and when the posterior force is increased from 0 to $-250 \mathrm{~N}$. These regions of interest for the cyclic curves are highlighted in Fig. 5. Under these particular loading conditions, predictions were in good agreement with the displacements recorded by the simulator for both designs and flexion angles.

Incorporating friction between the TKR components produced a region of no displacement when the magnitude of the A-P force is less than $100 \mathrm{~N}$. Similar observations were made with Sathasivam model (Figs. 3 and 4) and in the experimental cyclic tests (Fig. 5), where the flat line at the top and bottom of the curves showed that there was no displacement between the TKR components when the force direction changed.

\subsection{Influence of varying soft tissue restraints with flexion angle}

Fig. 6 shows the force-displacement curves for the Kinemax Lowstress and Kinemax Condylar TKR designs, with friction and with constant and varying soft tissues restraints. For both designs, modelling the soft tissue restraints using constant stiffness leads to the underestimation of both anterior and posterior laxity of the TKR joint. Constant stiffness leads to underestimation of tibial tray total A-P displacements at 10 and $60^{\circ}$ flexion 3.5 and $5 \%$, respectively for the Kinemax Lostress TKR, 6 and $8.5 \%$ for the Kinemax Condylar TKR. The main differences between the results obtained with constant or varying stiffnesses were observed in the evaluation of the anterior displacements of the tibial ray as shown in Fig. 6. 

$10^{\circ}$ flexion

Kinemax Lowstress $60^{\circ}$ flexion

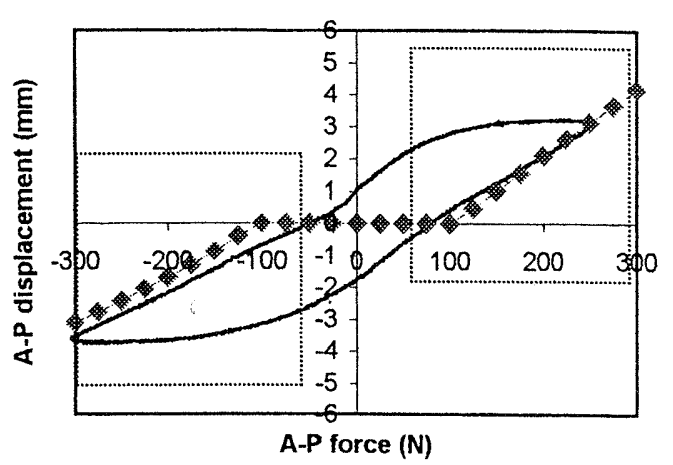

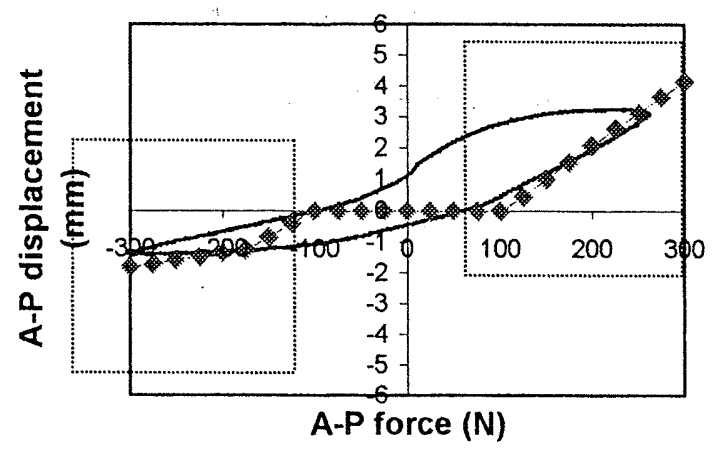

Kinemax Condylar
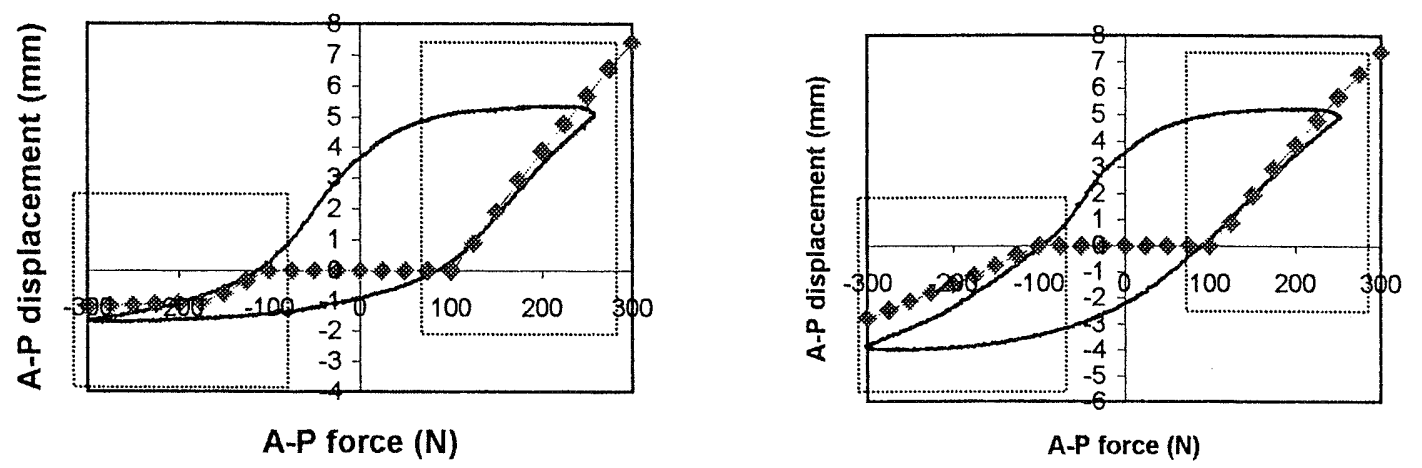

Fig. 5. A-P laxity results for the Kinemax Lowstress and Kinemax Condylar TKR designs predicted by $(\bullet) \mathrm{I}-\mathrm{DEAS} \mathrm{S}^{\mathrm{TM}}$ with friction coeff $\mu=0.0^{\circ}$ measured by (-) Walker knee simulating machine (Sathasivam and Walker, 1997).

\section{Discussion}

The close agreement between the experimental data reported in the literature and the results obtained with the model leads to the conclusion that such a model provides an accurate description of the kinematics of TKR in the sagittal plane, for the different geometries, loading conditions, and soft tissue restraints. Despite the assumptions made in the model, such as rigid-body behaviour and $2 \mathrm{D}$ motions, the good agreement between the different results demonstrates the value of this approach.

Soft tissues (ligaments and capsule) play a fundamental role in the intact knee joint in restraining the relative anterior-posterior motions between the femur and the tibia (Crowninshield et al., 1976; Fukubayashi et al., 1982; Gollehon et al., 1987; Beynnon et al., 1996). In order to evaluate TKR kinematics realistically, the action of soft tissues should be taken into account and introduced into the model of the knee joint. Sathasivam and Walker (1997) modelled the soft tissue restrainsts by non-linear springs. However, they did not take into ac- count that soft tissue restraints vary with flexion a: The model described in this paper presents prelimi results of the effect of modelling soft tissue as non-li springs with stiffness that vary with the flexion ang] was shown that using constant soft tissue restraints 1 to underestimate the A-P laxity, in particular for conforming designs, at $60^{\circ}$ flexion. However, only a r imum of $10 \%$ variation was obtained for total AP placements predicted with constant or varying soft $\mathrm{ti}$ restraints. It was therefore concluded that the effer varying soft tissue restraints with flexion angle is nc significant as it might be expected, at least for the fle angles examined.

The model presented in this study represents one o very few attempts of using virtual prototyping soft for modelling biomechanical systems. The model developed without any requirement for computer gramming, thus allowing rapid progress towards $v$ able results. The model can be readily utilised by o researchers and parameters such as TKR geometry. ternal loading conditions or soft tissue restraints cal easily changed. Additionally, once the input param $\epsilon$ 




\section{References}

Beynnon, B., Yu, J., Huston, D., Fleming, B., Johnson, R., Haugh, L., Pope, M.H., 1996. A sagittal plane model of the knee and cruciate ligaments with application of a sensitivity analysis. Journal of Biomechanics Engineering 118, 227-239.

Blunn, G.W., Joshi, A.B., Minns, R.J., Lingren, L., Lilley, P., Ryd, L., Engelbreck, E., Walker, P.S., 1997. Wear in retrieved condylar knee arthroplasties: a comparison of wear in different designs of 280 retrieved condylar knee prostheses. Journal of Arthroplasty 12, 281-290.

Crowninshield, R.D., Pope, M.H., Johnson, R.J., 1976. An analytical model of the knee. Journal of Biomechanics 9, 397-405.

Essinger, J.R., Leyvraz, P.F., Heegard, J.H., Robertson, D.D., 1989. A mathematical model for the evaluation of the behaviour during flexion of condylar-type knee prosthesis. Journal of Biomechanics 22, 1229-1241.

Fukubayashi, T., Torzilli, P.A., Sherman, M.F., Warren, R.F., 1982. An in vitro biomechanical evaluation of anterior-posterior motion of the knee. Journal of Bone and Joint Surgery 64A, 258-264.
Garg, A., Walker, P.S., 1990. Prediction of total knee motion using a three-dimensional computer graphics model. Journal of Biomechanics 23, 45-58.

Gollenhon, D.L., Trozilli, P.A., Warren, R.F., 1987. the role of the posterolateral and cruciate ligaments in the stability of the human knee. Journal of Bone and joint Surgery 69, 233-242.

Knutson, K., Lewold, S., Robersson, O., Lidgren, L., 1994. The Swedish knee arthroplasy register - a nation-wide study of 30,003 knees 1976-1992. Acta Othopaedica Scandinavica 65, 375-386.

Luger, E., Sathasivam, S., Walker, P.S., 1997. Inherent differences in the laxity and stability between the intact knee and total knee replacement. The Knee 4, 7-14.

Sathasivam, S., Walker, P.S., 1997. A computer model with surface friction for the prediction of total knee kinematics. Journal of Biomechanics 30, 177-184.

Walker, P.S., Blunn, G.W., Broome, D.R., Perry, J., Watkins, A., Sathasivam, S., Dewar, M.E., Paul, J.P., 1997. A knee simulating machine for performance evaluation of total knee replacements. Journal of Biomechanics 30, 83-89. 
